\title{
COMPETENZA COMUNICATIVA INTERCULTURALE: ALCUNI ESEMPI DI INTERAZIONI PLURILINGUISTICHE IN ISTRIA
}

\author{
Rita Scotti Jurić \\ Università Juraj Dobrila di Pola \\ Dipartimento di studi in lingua italiana \\ Davorin Brajković \\ Università Juraj Dobrila di Pola \\ Facoltà di Filosofia
}

\section{Riassunto}

In base ad una ricerca sulla comunicazione plurilingue e interculturale nell'area plurilingue dell'Istria, il saggio analizza le interazioni verbali dei giovani bilingui di madrelingua italiana. Dopo una breve descrizione degli studi sul bilinguismo e sul multiculturalismo nell'area istroquarnerina, vengono esaminate le principali motivazioni che portano all'uso di code-switching e ad interferenze linguistiche durante le interazioni informali dei parlanti. Esempi di conversazioni, registrate in situazioni familiari, ed altre tratte dai social network, verranno discussi per dimostrare che l'analisi della conversazione è in grado di fornire una visione significativa della comunicazione interculturale e plurilinguistica. Si cercherà di spiegare, in particolare, come la scelta della lingua e l'alternanza del linguaggio siano in grado di soddisfare gli scopi comunicativi impellenti e come una competenza limitata nella terza e quarta lingua può essere sfruttata nella comunicazione interculturale.

Parole chiave: Competenza interculturale, comunicazione interculturale, competenza plurilingue, discorso bilingue, code-switching, interferenza, funzioni linguistiche. 


\section{Competenza plurilingue e discorso bilingue}

La comunicazione interterculturale, quale insieme integrato di abilità e facoltà generali, include anche la conoscenza di particolari linguistici che arricchiscono la relazione comunicativa, come gli stili di comunicazione e le regole di interazione. Il successo della comunicazione interculturale fra appartenenti a culture diverse dipende, in modo particolare, dalla scelta del code appropriato che viene usato come mezzo di comunicazione usuale tra interlocutori diversi. Usare un code comune non significa soltanto utilizzare la stessa lingua, ma condividere anche alcune intenzioni comunicative. E possibile che un contatto linguistico quotidiano renda deboli i confini linguistici di ciascuna lingua e si giunga a delle mescolanze linguistiche, culturali e comportamentali, alle volte persino identitarie. Le ricerche sul linguaggio dei parlanti bilingui e plurilingui evidenziano due correnti teoriche contrastanti. La prima vede nel contatto linguistico grandi possibilità di mescolanza di lingue che sottovaluta la condizione di bilinguismo, ritenuto “zoppo" (Nelson 1982; Mc Namara, 1967). Secondo queste ideologie dell'interculturazione, il bilinguismo eserciterebbe un'influenza negativa sullo sviluppo mentale del parlante, a maggior ragione se ancora bambino. Ne deriva un'interpretazione negativa delle condizioni mistilingui del parlante che porta al pregiudizio dello svantaggio linguistico, dovuto appunto all'inadeguato approccio valutativo deficitario della sua competenza linguistica. La seconda corrente teorica (Mc Laughlin, 1984; Peale Lambert 1962; Cummins 1984), sotto l'influenza degli studi sul linguistic contact rivaluta il campo di lavoro del bilinguismo, sostituendo alla nozione di interferenza quella più positiva di trasferimento (o transfert) linguistico. Gli studi sul linguaggio dei parlanti italofoni dell'Istria croata e del Quarnero (Milani Kruljac, 1990, 2003; Scotti Jurić, 1998, 2003, 2010) rilevano la presenza di una competenza bilingue che implica la capacità di passare dalla lingua di casa alla lingua di scuola, dalla lingua della strada alle lingue specialistiche con manifestazioni di intelligenza e creatività linguistica. Pur lamentando delle preoccupazioni, i ricercatori concordano nel non veder niente di anormale nella loro competenza plurilingue, adeguata alle esigenze e agli usi della comunità in cui vivono, se per competenza plurilingue e pluriculturale intendiamo l'abilità «di usare le lingue per intenti comunicativi e per partecipare a interazioni interculturali nelle quali la persona, in qualità di agente sociale, ha la competenza di varie lingue a livelli differenti, ed esperienze in varie culture.» (Trim et al., 2001: 16, tr.d.a.). Ciò significa che gli individui dovrebbero costruire una competenza comunicativa integrale in cui lingue e culture diverse si fondano in un repertorio linguistico più completo e aggiornato, attraverso un discorso plurilingue fruibile e coerente (Ludi e Py, 1986). Il loro comportamento verbale dovrebbe essere la prova di una competenza plurilingue comunicativa in azione, cioè di una capacità di individuare i tratti costitutivi degli eventi comunicativi plurilingui che 
permetta di passare da una lingua all'altra in base alla situazione comunicativa creatasi. Si tratta di mettere in uso tutte le risorse pragmalinguistiche, ed in primis il saper fare, ossia saper usare la lingua in situazioni comunicative reali (Balboni, 2002). Il discorso plurilingue che si viene a creare non è il risultato di più competenze monolingui, né esso è segno di una competenza linguistica disabile, invalida nelle forme e nelle strutture, in quanto composta da diverse conoscenze estranee alle singole norme monolinguistiche: esso è piuttosto un uso creativo e pragmatico delle lingue conosciute e parlate, con l'intento di ottimizzare le risorse cognitive e adattarle alle nuove esigenze comunicative (Trim et al., 2001). Nei parlanti plurilingui le competenze in ciascuna lingua sono diverse, e soprattutto non sono né uguali né necessariamente simili a quelle dei parlanti monolingui in quanto svolgono una serie di funzioni diverse, a seconda delle diverse esigenze di comunicazione. I passaggi tra i diversi sistemi linguistici, ad esempio, possono assumere maggiore importanza in certi atti comunicativi e meno in altri (Coste, Moore, Zarate, 2009). Per questo motivo le competenze di un individuo plurilingue sono in larga misura complementari, in quanto non si sovrappongono completamente nelle diverse lingue, e l'uso alternato di componenti di diversi repertori è una questione di sviluppo strategico della competenza comunicativa. Il tratto più comune della competenza plurilingue è sì uno stato di squilibrio, ma è allo stesso tempo un tratto complesso e dinamico, e lascia spazio a fenomeni originali, come lo è il discorso bilingue.

Questo insieme di risorse linguistiche diventa quindi un capitale linguistico ed opera a seconda della situazione e dell'interlocutore: in ambienti monolingui il bilingue può scegliere di nascondere una parte del suo repertorio linguistico per evitare di essere percepito come differente, in situazioni plurilingui può invece mobilitare tutte le sue lingue al fine di selezionare i suoi interlocutori, includerli o escluderli dalla conversazione, modificare il livello del discorso, parlare con maggiore efficacia, citare altri partecipanti, e via dicendo.

Il discorso bilingue è caratterizzato da un frequente uso di interferenze e commutazioni di codice o code switching, cioè da fenomeni di cambio della lingua adottata nell'interazione. La commutazione di codice è sempre frutto della decisione del parlante di cambiare la lingua del discorso e di continuare la conversazione in un'altra lingua. In questo senso essa si differenzia dall'interferenza, in quanto non prevede nessuna sovrapposizione di elementi linguistici appartenenti ai due codici diversi; al contrario, anzi, essa costituisce un cambiamento conscio e voluto nel corso dell'interazione comunicativa. La commutazione è motivata da una strategia pragmatica, con scopi interazionali, ed è quindi un fenomeno del discorso, mentre l'interferenza comporta una confluenza dei due sistemi linguistici ed una giustapposizione di essi. Gli studi 
sullinterferenza delle lingue croata e italiana hanno evidenziato i punti morfosintattici e semantici critici dell'enunciato in cui essa avviene (Milani, 1990; Scotti Jurić, 2003). L'indagine che presentiamo si allinea agli studi di pragmalinguistica ed è volta ad esplorare le possibili motivazioni che causano il code switching. Tra le molte tipologie proposte per una categorizzazione funzionale della commutazione di codice segnaleremo quella di Wardhaugh (1995) che distingue due tipi principali di code switching, motivati l'uno dal cambiamento dell'interlocutore e delle circostanze esterne del discorso, e il secondo motivato dal cambiamento dell'argomento del discorso. Appel e Muysken (1987) propongono le categorie del code switching basate sul modello funzionale di Jakobson (1976) e Halliday (1983, cioè sulle funzioni referenziale, direttiva, espressiva, fàtica, metalinguistica e poetica. Gumperz (1964) distingue due tipi principali di code switching: transazionale, quando avviene al modificarsi dei componenti del discorso (l'argomento, $\mathrm{i}$ partecipanti, il luogo) e dipende quindi dalla situazione, e metaforico, quando il parlante decide automaticamente di inviare il messaggio codificandolo meddiante una delle opzioni comunicative di cui dispone, indipendentemente da cambiamenti situazionali. Qui è la commutazione stessa, in quanto scelta deliberata e non condizionata da fattori esterni, a farsi portatrice di significato. Comunque sia, è chiaro che la commutazione di codice è legata ai tipi di atti linguistici messi in gioco, e più precisamente alle sequenze linguistiche o mosse di cui essi sono composti. ${ }^{[1]} \mathrm{E}$ visto che le parti degli atti linguistici sono facilmente „trasportabili“ da una lingua all'altra (oda un dialetto all'altro), esse diventano tasselli indispensabili per potenziare o semplicemente mantenere vivo un discorso, una funzione linguistica prescelta nella comunicazione in corso (Scotti Jurić, 2003).

\section{Metodologia della ricerca}

Nella nostra ricerca siamo partiti da alcune ipotesi, alcune parzialmente già sottoposte a verifica nelle ricerche precedenti (Scotti Jurić, 2003):

I1: i parlanti sono pienamente consci della competenza linguistica dei loro interlocutori abituali, per cui si rivolgano nella loro lingua;

I2: nelle situazioni conversazionali tra plurilingue e monolingue il plurilingue non si avvale della possibilità di cambiare il codice linguistico;

I3: nelle situazioni conversazionali tra plurilingui con competenze linguistiche parificate

[1] Gli atti appartenenti alla stessa tipologia sono, poi, parti costituenti di una funzione linguistica. 
vengono messe in atto tutte le possibili capacità espressive nei vari codici linguistici posseduti;

I4: esiste un code switching obbligatorio, imposto come norma comportamentale, legato alla presenza di monolingui nella conversazione dei bilingui;

I5: esiste un code switching non strettamente obbligatorio tra bilingui, un fenomeno comunitario che non ha regole precise;

I6: ciononostante se ne possono individuare le motivazioni soggiacenti. E su quest'ultima ipotesi che ci si soffermerà maggiormente in questa ricerca: accanto alle regole funzionali della commutazione che presenteremo, è importante dire che non sempre il passaggio da un codice ad un altro ha una motivazione o una funzione consapevole, soprattutto per quanto riguarda quelle commutazioni che avvengono all'interno di una stessa frase. Nei casi in cui la commutazione avviene senza motivi apparenti parleremo di categoria "vuota".

Per avere uno strumento di indagine chiaro e circoscritto, abbiamo creato, ai fini della presente ricerca, una griglia con le tre principali funzioni del linguaggio: la funzione interattiva legata ai partecipanti, la referenziale legata all'argomento e quella emotiva legata al messaggio. Di seguito segnaliamo per ciascuna di tali funzioni le situazioni, gli atti o le mosse linguistiche in cui esso può aver luogo.

Tabella 1. La funzione interattiva

\begin{tabular}{|c|c|}
\hline Fint 1 & interiezione, quando un'esclamazione viene prodotta nell'altra lingua \\
\hline Fint2 & inserzione di intercalari, cioè di elementi riempitivi \\
\hline Fint3 & $\begin{array}{l}\text { specificazione del destinatario del messaggio oppure rinegoziazione del codice in } \\
\text { uso nel caso dell'interlocutore bilingue: quando il cambio di codice è motivato dalla } \\
\text { scelta di una data lingua per rivolgersi ad un destinatario in particolare }\end{array}$ \\
\hline Fint4 & $\begin{array}{l}\text { segnale del cambio di interlocutore; quando interviene ad esempio una terza } \\
\text { persona in uno scambio, il parlante può cambiare codice per coinvolgerla e riferirsi } \\
\text { direttamente ad essa, o viceversa per escluderla dal discorso e non farsi comprendere }\end{array}$ \\
\hline Fint5 & presenza di atti linguistici particolari quali saluti ed allocutizioni \\
\hline Fint6 & segnali discorsivi che connettono elementi frasali, interfrasali ed extrafrasali \\
\hline
\end{tabular}


Tabella 2. La funzione referenziale

\begin{tabular}{|l|l|}
\hline Fref1 & $\begin{array}{l}\text { qualificazione del messaggio: quando il bilingue impiega un'altra lingua per } \\
\text { commentare ulteriormente o puntualizzare quanto detto; chiarimenti; prese di } \\
\text { distanza da ciò che viene detto }\end{array}$ \\
\hline Fref2 & $\begin{array}{l}\text { segnale del cambio di argomento o di tipo di discorso, che può passare ad esempio } \\
\text { da un modo formale ad uno informale; ricollegamento ad una conversazione } \\
\text { precedente interrotta e poi ripresa }\end{array}$ \\
\hline Fref3 & soluzione di un problema di accesso al vocabolario \\
\hline Fref4 & marchio di appartenenza di un bilingue entro il gruppo dei pari della comunità \\
\hline
\end{tabular}

Tabella 3. La funzione emotiva

\begin{tabular}{|c|c|}
\hline Fem1 & $\begin{array}{l}\text { personalizzazione/oggettivazione: riflesso del coinvolgimento o distanziamento } \\
\text { emotivo del parlante rispetto all'argomento del discorso o ai partecipanti } \\
\text { all'interazione comunicativa }\end{array}$ \\
\hline Fem2 & inclusione/esclusione dei parlanti monolingui presenti dal discorso \\
\hline Fem3 & $\begin{array}{l}\text { distinzione tra discorso diretto e riferito: si tratta di citazioni, quando il parlante } \\
\text { riproduce in lingua originale e sotto forma di discorso diretto il contenuto di un } \\
\text { messaggio a scopo persuasivo oppure per rendere al massimo l'espressività della } \\
\text { frase riferita }\end{array}$ \\
\hline Fem4 & $\begin{array}{l}\text { uso poetico o scherzoso: quando si vuole dare un tono enfatico ad un particolare } \\
\text { commento, creare una certa "complicità" con l'interlocutore }\end{array}$ \\
\hline Fem5 & sfruttamento del potenziale connotativo di certe parole \\
\hline
\end{tabular}

In questo saggio presenteremo l'analisi di un caso di registrazione di un parlato spontaneo e di alcuni estratti di chat di due giovani italofoni ( $\mathrm{F}=19$ anni e $\mathrm{C}=18$ anni). La registrazione (durata: $21^{\prime} 28$ ") è stata effettuata in famiglia e riprende una situazione di interazione tra due studenti impegnati a consultare i siti web universitari per informarsi sul prossimo esame di ammissione. I dati sono stati analizzati con il software MAXQDA 11. La lingua di comunicazione è l'istroveneto (LM), ma chiaramente vi si intrecciano parole croate (L2), inglesi (L3) e tedesche (L3), come pure si notano chiare infiltrazioni di parole italiane standard, sentite come un codice diverso, alla pari del croato. I testi ripresi da Facebook sono stati analizzati con le stesse modalità del testo registrato e poi trascritto, con l'intento di capire le motivazioni che sottostanno al cambiamento di codici linguistici. Nell'analisi dei due corpus non abbiamo perso di vista il fatto che il meccanismo che regola l'avvicendamento dei turni e che spiega le proprietà osservate è un insieme di regole preordinate che operano turno per turno per cui possiamo 
trattarlo come un sistema a gestione locale. Ne deriva una conseguenza significativa: il sistema locale fornisce una motivazione affinché i partecipanti ascoltino e analizzino ciò che viene detto, specie in una comunicazione con prevalenza di funzioni regolative e referenziali.

\section{Analisi e discussione}

\section{Funzioni del code switching}

Le registrazioni presentamo caratteristiche funzionali e pragmatiche motivate dal cambiamento di codice, spesso accompagnato da interferenze strutturali come quella morfosintattica (IMS) che ricalca un ordine delle parole non canonico nella lingua italiana $\mathrm{S}+\mathrm{P}+\mathrm{C}$, ma quello più libero della lingua croata (Qua tutto quanto ti devi scriver; questo tutto ti mandi, qua solo el programa ti meti, ti devi due canzoncine sonar). Abbondante è l'uso di sostantivi (prijavnica, predmet, glavni predmet) con i quali l'interlocutore risolve un problema di accesso al vocabolario della lingua italiana, visto che la lingua vissuta nella sua quotidianità sociale è appunto quella croata con oscillazioni nelle parole di uso settoriale in croato (prijemni e prijamni). L'interiezione To! sta ad indicare la presenza di un'altra funzione che determina il mutamento del codice, quella interattiva o interpersonale.

C Bon, per iniziar ti pol guardar ti questo folder qua.

C
To! (Fint1)

Neanche dir! ((pausa)) Cossa verxo qua?

Questo qua, ((pausa)) hmm, questo...te xe...la prijavnica (Fref3)

E questo te xe el programa de quel, hm de la roba che ti sonerà, 'nači (Fint2) verxi questo. Qua tutto quanto ti devi scriver (IMS), nači, (Fint2) el studio, cos che ti vol, bla, bla, bla...((pausa)) e la data, bla, bla, questo tutto ti mandi (IMS).

(2)

Qua ti ga prob list, (Fref3) 'nači (Fint2) qua solo el programa ti meti (IMS).

Questo xe per...'Nači, (Fint2) quando che la ti scegli el programma questo ((incomprensibile)) bla, bla, bla

Hochschule für Musik. Hochschule für Musik (Fem3).

Questo xe el programma per ((pausa))

per el prijamni (Fref3)

per el prijemni (Fref3) o per un/qualche z... ok, va ben. 
C

Ti/ Solo una ti ga fato povečat.(IMS) ((pausa)) questo uopće (Fint2) no ti ga guardà. ((pausa)) E! e ti sa cossa che ancora i te domanda?

C Se no ti iscrivi piano come glavni predmet (Fref3), ti devi sonar. Se ti scrivi qualche altro predmet (Fref3) ti devi sonar el piano.

C Ti devi due canzoncine sonar, (IMS) dipendi. Un Bach e ancora qualcosa, quel che ti gavevi xà (IMS).

La maggior parte dei code switching individuati, come quelli degli estratti che seguono, avvengono in funzione referenziale in quanto il testo registrato è fortemente regolativo, informativo e rappresentativo. Ci sono però cambiamenti di codice motivati da funzioni interattive, e contrassegnati dall'uso di intercalari ('nači: abbreviazione di 'znači', mislim: oppure la sua abbreviazione 'mis'), interiezioni (Insomma, to ti je tako; Uglavnom go finido) e frasi esclamative che rafforzano il collegamento interattivo tra $\mathrm{i}$ due interlocutori (Ma da ne bil; Ni moguće).

C

$\mathrm{F}$

C

$\mathrm{F}$

C

$\mathrm{F}$

C

(5)

Questo te xe un primjer (Fref3) de solfeggio ((pausa)) ...'Nači, (Fint2) cos' che i ne domanderà de solfeggio ((pausa)) Hmm...

Solfeggio...?

No, no, speta questo xe... Si, solf... 'nači, (Fint2) quel che i te domanda nel...

Questo ti devi cantar? Che diavolo xe sta roba...? ((pausa))

No so. ((pausa)) Akord diktat (Fref3) ti vedi...

Ma da ne bi! (Fit1) aha!

Si. Questo, no so... Questo xe teoria, valjda, (Fint2) me par. Insomma, to ti je tako (Fint 2).

$\mathrm{F}$

C

C

F

C

C

Melodische, (Fref3) ara qua, questo sarà meloritamski (Fref3) ((pausa)) No, penso de no.

Però questo me par che xe teorija (Fref3) o no so, deso sera questo, misl... (Fit2) calilo xo ((pausa)) ti ga ancora uno de solfeggio.

Ni moguće! (Fint1), 'peta... ((incomprensibile)) questo xe... ((pausa)) To! (Fint1), la. ...Ritam (Fref3), ...el...

Cosa ... no me ricordo qualcoss... ((pausa))

ritam (Fref3) ti vedi! Uglavnom (Fint2), ti ga finido. 
Negli estratti che seguono, oltre ad altri esempi di interferenza, si notano un caso di commento (pisanje nota $u$...u ključevima), ed un segnale di cambio di codice per coinvolgere il terzo parlante, quello virtuale rappresentato dal computer, e riferirsi direttamente ad esso, come se si volesse includerlo nella conversazione ( $J a$ !, in tedesco invece di Sì! in italiano).

C

$\mathrm{C}$

$\mathrm{F}$

C

(7)

C

$\mathrm{F}$

$\mathrm{C}$

$\mathrm{F}$

$\mathrm{F}$

F

$\mathrm{C}$

F

(8)

((incomprensibile)) crtovlje (Fref3). E go guardado ((pausa)) sulla pagina dell'accademia de Zagabria. I domanda la stessa roba. 'Nači... (Fint2) ((pausa)) sull'accademia (IMS). El prim/el numero uno iera questo...nači (Fint2) crtovlja (Fref3) e sta roba qua..

'Nači (Fint2) pisanje nota u ...u ključevima (Fint4).

$\mathrm{Hm}$ !

questo o ad esempio pisanje nota (Fref3) tipo ad esempio c mali.

Questo saria intervali? (Fref3) ((pausa)) Dovessi esser...

Sì, velika septima! (Fint1)

$\mathrm{Hm}$ ! Peta che torno...((pausa))

((pausa)) t'vedi klein... (Fref3) no, mala sep...(Fref3) klaine...

Kleine (Fref3)

Ja. (Fem4)

((pausa)) Hm ((pausa)) e cossa saria tuta sta roba...((pausa))

uzlazno i silazno, questo xe kao primjer (Fref3)

F

C

$\mathrm{F}$

C

F

C

$\mathrm{C}$

F

Ok ((pausa)) kleine, reine (Fref3)

No so cossa che xe sta roba, reine (Fref3) ((incomprensibile))

Hm... čista. (Fref3)

reine (Fref) dovesi esser čista (Fref3) ma no devi ...e übermälzige? (Fref3)

forsi xe...povečana! (Fref3) Oh, no! klein o über (Fref3)

smanjena! (Fref3)

Kao: kako seksta može biti smanjena ili povečana (Fint4)

Može, smanjena seksta, (Fint4) come no!

La citazione di singole parole nella L2/L3 oppure di un intero enunciato è una delle pratiche più vive e sentite dei parlanti plurilingui. L'espressione cantus firmus è 
inteso come un prestito, non come una commutazione di codice dal latino.

(9)

C

F

F

C

(10)

Scrivi: Hochschule (Fem3) Detmold.... ((pausa)) ((pausa))

Zero!

F

sto tuo computer xe...((pausa)) veloce come una sajeta! (Fem5)

F ((pausa)) What'ever (Fref2) ((pausa)) questa roba no se verxi.

M E cosa ti vol che ghe faso!

Niente, pianxemo!

Aaufiderzen! (Fint5)

I dialoganti si impegnano costantemente in un reciproco monitoraggio, comprendendo la voce virtuale del computer come un terzo interlocutore che lo si può includere dal discorso o escludere. L'intenzione è sempre quella di verificare che il proprio discorso sia compreso correttamente: l'enunciato Scrivi: Hochschule Detmold... è verificabile direttamente nel turno successivo quando l'ascoltatore, ora divenuto locutore non sfrutta la possibilità di prendere il turno che gli spetta (come in questo caso, in cui si avvale di una lunga pausa di riflessione). Il primo parlante verifica a questo punto l'interpretazione dell'altro (Zero!). Nel nostro caso il primo interlocutore spiega il motivo del suo indugio: Sto tuo computer xe...((pausa)) veloce come una sajeta! La traduzione della frase Schreiben sie zu folgender Liedmelodie ein Ober-oder Unterstimme viene fatta in italiano standard ricalcando la funzione metalinguistica tante volte usata nei banchi di scuola: Scrivete sulla seguente melodia una voce superiore o una inferiore. 


\section{Le lingue e la frequenza d'uso}

Le parole dell'italiano standard usate dai due interlocutori ${ }^{[2]}$ provengono da reminiscenze scolastiche o culturologiche dei più svariati settori: citazioni di frasi (come "la torre di Pisa sta crollando"), titoli di film (Matrimonio a quattro mani), cibo italiano (panettone), espressioni eloquenti (la società scientifica), modi di dire, fraseologia (sei tu il capo delloperazione, il genio del computer), terminologia specialistica (solfeggio, esame di ammissione), parole italianizzate (per iniziar).

$\begin{array}{ll}\text { F } & \text { No sta magnar adesso che lavoremo. } \\ \text { C } & \text { Hmmm ((pausa)) La torre di Pisa sta crollando (Fem4). } \\ \text { F } & \text { Ti te diverti con le fette de panettone! (Fref3) ma sii seria. } \\ \text { C } & \text { Ma scusa, sei tu il capo delloperazione (Fem4). }\end{array}$

Numerose sono le interferenze dal croato, in particolare le espressioni legate alla funzione comunicativa e interattiva, sotto forma di intercalari, riempitivi, modi di dire, stampelle linguistiche, e via di seguito. Le parole croate registrate in questa ricerca sono state le seguenti: la prijavnica, 'nači, sto posto, prijemni, prijamni, valjda, in D dur, ritam, fis, cis, fis uno, crtovlja, pisanje nota u ključevima, c mali, velika septima, mala septima, mala terza, uzlazno i silazno, primjer, kako seksta može biti smanjena ili povećana, može, miksolidijski, crkveni tonaliteti, polifonija, ti ga fato povećat, uopće, glavni predmet, suspregnut ću se, to je to, fa strajk, u stilu, neka, jeste li mislili, glava, dom, diplomski, preddiplomski, pa neka, to nije to. La lingua inglese che per molti versi non si sente neanche più come una lingua straniera ricalca molte espressioni ormai radicate profondamente nella lingua dei giovani comead esempio quelle inerenti il computer con adattamenti morfologici in istroveneto (participio passato: connettado): folder, desktop, internet, computer, google, campus, examination, student portal, master, date, admission, program, home page, application form. Ci sono poi intere frasi inerenti la professione del musicista (only for master program for concert), intercalari o riempitivi come Whatever, e le insostituibili particelle di approvazione come yes e $O k$. Le parole tedesche appartengono alla terminologia specialistica degli studenti in questione: Hochschule für Musik, Akorddiktat, melodische, kleine Septima, eine, über, unter, oder, Sommer, Winter, Detmold, anmelden.

Il parlato dei due studenti comprende particelle ed elementi lessicali dei quali non è sempre chiaro lo status funzionale, in quanto si tratta di categorie che

[2] Queste parole non sono tutte visibili dagli estratti trascritti e presentati in 4.1.1., ma sono presenti nel discorso integrale registrato. 
appartengono al livello „tematico“ o „informazionale“ dell'enunciato: cioè, ehm, vedi, 'nači, 'sto, 'ara, e via dicendo. Sono categorie analitiche diverse da quelle elaborate dalla grammatica frasale che riterremo elementi discorsivi che valgono come riempitivi o connettivi pragmatici. Come in tutte le situazioni di un parlato spontaneo il corpus analizzato palesa una grande frequenza di pause, interruzioni ed autocorrezioni, lapsus, deittici, intercalari (speta, peta; valjda, sto posto). L'inglese ed il tedesco non presentano stampelle linguistiche dato che non incidono nel tessuto organico della comunicazione ma vi entrano con format individuali che non si integrano con la lingua.

\section{Competenza interculturale}

Tschirner suggerisce che la pratica multimediale è ottima per l'apprendimento delle lingue straniere: "Sprachen lernt man Multimedial. Man hört die Menschen, mit denen man sich unterhält, man sieht Ihre Lippen, ihre Mimik, ihre Gestik, und man sieht vielleicht sogar, worauf sie sich beziehen." (Tschirner, 1997, 55). Al computer le informazioni possono essere gestite seguendo percorsi scelti autonomamente e presentate in una sequenza non lineare secondo una modalità di tipo associativo, vicina al modo in cui l'essere umano organizza il proprio pensiero ed apprende le proprie conoscenze. In questo senso anche la comunicazione in forma scritta delle chat e di Facebook diventa una fonte preziosa per delineare il quadro della competenza plurilingue ed interculturale dei giovani locutori. La competenza linguistica interculturale di questi formati sembra sbizzarirsi maggiormente, non essendo legata ad un argomento fisso come nell'interazione registrata in ambito familiare analizzata sopra.

F. Ah kradeš!! (Fit1) niti ne znaš odkud je taj citat! (Fref4) aww ajde ajde (Fit1) Danke sehr liebe Schwester! (Fem1).

C. liebe Schwester (Fem3) samo to lajkam!(Fref4 )

F. hmm knjiga mudih izreka (fref4)!!!!!

C.Tantiiiiii auguriiii the happiest (and silliest) Happy Birthday song, which I love!!! (Fem4)

Pur non essendo la loro lingua madre, la lingua usata è il croato, scelta dettata dal mezzo per comunicare, cioè Facebook, uno strumento di interazione accessibile 
all'intera comunità di amici. In interessante code switching con atti linguisici in lingua tedesca da parte di F (reidente in Germania) ed in inglese da parte di C (residente in Croazia), testimonia il desiderio di interculturalità. Nell'enunciato 13 gli enunciati sono brevi, scambi di batture originali, fresche, divertenti e dove il punto forza è appunto il mutamento di codice controllato e gestito:

\author{
F. Danke seka! (Fem1) \\ C. To se inače pise u inbox, (Fref1). \\ F. Dont care C. (Fit2)
}

Diversa ancora è la comunicazione fatta tra di due giovani attraverso Skype, più privato e cancellabile in ordine di tempo, per cui non lascia traccia, non è visibile a persone estranee e garantisce una certa libertà di espressione. I due ragazzi comunicano in istroveneto (14) con mutamenti di codice in croato, insostituibile quando si tratta di riprodurre un dialogo diretto, oppure in atti linguistici appartenenti alla funzione interpersonale in ambito extra-familiare, nella società croatofona:

F. Ma che stronzo sto lik.(Fit5) Ne sekiraj se previse, i najbolima se dogadja! (Fit1) btw (Fem1) cossa ti vol che te rispondo sul Face? (Fref3) una volta co gavevimo ciapado 1 nagrada (Fref3) col trio no semo rivadi al zavrsni (Fref3) per un per de punti... e mi ge do dito a P.: ne brini P., drugi put ćemo rasturiti. (Fem3) e lui me ga dito: ali F. mi smo i ovaj put rasturili! (Fem3) Poanta (Fref3): no cori becar 5 da dokazes da si dobar Fit1).

C. Grazie. Ti me ga fato tanto oraspolozit. (IMS) guarda che anche ti certe volte ti sa esser romantican (Fref3). Cosa ti fa ti? Mi studio psihologija (Fref3). Quando ti me mandi le note unatoc mojoj trenutnoj totalnoj demotivaciji? (Fref1) Oggi go visto N. Che faca.(Fit5) Opet mi je digo zivac.(Fit5) Lo go mandado u rodni kraj! (Fit5). Ma nol ga sentido la destinazion. Te saluda la mia gazdarica (Fref3). Nox (Fit5).

Oltre che per sottolineare l'argomento, il referente, i problemi di vocabolario, il bisogno di commutare il codice è vivo per trovare nuove espressioni interattive valide, onde valorizzare meglio la propria personalità, l'identità, la specificità, la creatività, la destrezza. Su questa linea si articola anche la volontà di colorare il messaggio con espressioni emotivamente plastiche: potenziali connettivi diversi, usi scherzosi e poetici 
di parole, toni enfatici e particolari. Anche nella comunicazione tramite Skype troviamo interferenze morfosintattiche (IMS) che possono riguardare il lessico e la sintassi, come nel caso di "ti me ga fato tanto oraspoložit" dove si imprestano verbi, che vengono trattati come "voce del verbo fare + infinito del verbo in croato". Di questo tipo sono i costrutti come questi: far šrajbati; far cugnuti. L'interferenza lessicale (IL) è pure presente nel parlato dei due giovani essendo il lessico una categoria estremamente aperta e che, nel passaggio da un codice all'altro, incontra una resistenza minima. È soprattutto il croato ad influenzare il discorso in italiano con parole che trasmettono i significati relativi alle esperienze e ai concetti tipici di un dato ambiente sociale.

- Andemo cuzamen stasera? (avverbio di modo'zusammen:)

- Šprehali (dal ted. sprechen) smo dva sata, i to sam ja njega zvao.

Singolare è il fatto che su Skype il code switching interessa enunciati completi e circoscritti, offrendo ritagli di comunicazioni autentiche in lingua croata e prese dal vivo.

In chiusura di questo paragrafo daremo un esempio di ciò che avviene nella comunicazione tra un bilingue e un monolingue. Quando F e C non parlano tra di loro, ma con un interlocutore monolingue croatofono che non comprende l'italiano, il loro linguaggio non è più plurilingue, ma bilingue. Sparisce la lingua italiana che fuori dall'ambiente istroquarnerino è ritenuta meno prestigiosa e rimane l'inglese come lingua più diffusa.

C. Dodji na skype. (Fref3)

H. Dobro dakle, sutra mi je exam (Fem4) iz harmonike... nakon toga haj lajf (Fem5) btw (Fit6) gdje ti je braco ???

C. Braco dolazi za 20tak dana javi se kad budes free (Fem5).

Rispetto alla comunicazione in lingua croata "di sottofondo" in lingua croata, traspaiono sostantivi molto frequenti nella comunicazione quotidiana locale. La comunicazione tra bilingue e monolingue non presenta casi di inteferenza, per cui la lingua di partenza non viene intaccata nella sua struttura sintattica, ma vi vengono semplicemente inseriti elementi semantici provenienti da unaltra lingua. La commutazione di codice è dettata da funzioni piuttosto emotive, dunque non necessarie, ma gratuite, creative, per il gusto di farlo. 


\section{Conclusioni}

Per le persone nell'ambito immediato del locutore, l'interferenza viene intesa e correttamente interpretata come una possibilità di garantzire una maggiore espressività, vivacità o precisione al linguaggio. A lungo andare si viene a creare una specie di "lessico familiare" in cui l'interferenza non è più sentita come tale, perché compresa e adottata prontamente dai bilingui che formano, col tempo, un "serbatoio" di lessico misto. I bilingui, una volta allenato l'orecchio, considerano queste interferenze più o meno normali, a seconda del loro grado di scolarizzazione, di cultura, di età e di motivazioni psicologiche socialmente modellate. La facilità di espressione verbale, accompagnata da una scarsa abilità nel tener distinte le lingue, dipende dalla risonanza che essi desiderano dare al testo prodotto. Il linguaggio degli interlocutori analizzati è caratterizzato da una competenza plurilingue che colora il loro discorso bilingue italo-croato, per cui essi riescono a passare da una lingua alle altre a seconda della situazione, nella stessa conversazione, a volte all'interno dello stesso enunciato. Il ricorso a usi strategici delle risorse linguistiche serve a negoziare significati, a mantenere la comunicazione aperta, a dare informazioni su chi parla, sulla sua identità sociale e culturale, a indicare il posto che occupa nella conversazione, o la natura dello scambio. La commutazione di codice nello stesso discorso non è un'indicazione dell'incapacità dei parlanti di distinguere le lingue singolarmente, bensì, il più delle volte, essa è piuttosto un indice della padronanza di tutti i sistemi in contatto, dove in una stessa sequenza alternativa, ogni lingua segue regole precise. Il linguaggio degli istroquarnerini abbonda di prestiti e di commutazioni volontariamente, coiè consciamente, controllate come dalla loro assenza, nel discorso dei bilingui in ambiente monolingue. 


\section{BIBLIOGRAFIA}

APPEL, R., MUYSKEN, P., 1987, Language contact and bilingualism, Edward Arnold, London.

CUMMINS, J., 1984, Bilingualism and Special Education: Issues in Assessment and Pedagogy, College Hill Press, San Diego.

COSTE, D., MOORE, D., ZARATE, G., 2009, Plurilingual and Pluricultural Competence, Studies towards a Common European Framework of Reference for language learning and teaching Language, Language Policy Division, Strasbourg.

GUMPERZ, J. J., 1964, Linguistic and social interaction in two communities, "American Anthropologist", 66(6), 2, 137-153.

HALliDAY, M. A. K., 1983, Il linguaggio come semiotica sociale. Un'interpretazione sociale del linguaggio e del significato, Zanichelli, Bologna.

JAKOBSON, R., 1976, Saggi di linguistica generale, Feltrinelli, Milano.

MC LAUGHLIN, B., 1984, Second Language Acquisition in Childhood, N Lawrence Erlbaum Associates, Hillsdale.

MC NAMARA, J., 1967, The bilingual's linguistic performance: A Psychological Overwiew, "Jornalnof Social issues", 23, 3.

MILANI, KRULJAC, N.,1990, La Comunità Italiana in Istria e a Fiume fra diglossia e bilinguismo, Centro di Ricerche Storiche di Rovigno, Rovigno.

MILANI, KRULJAC, N., 2003, Litaliano fra i giovani dell'istro-quarnerino, (a cura di) Pietas Julia-Edit, Pola-Fiume.

NELSON, C., 1982, Intelligibility and non-native varieties of English. In: KACHRU, B. (ed.), The other tongue: English across cultures, University of Illinois Press, Urbana, IL.

PEAL, E., LAMBERT, W. E., 1962, The Relation of Bilingualism to lntelligence, "Psychological Monographs", 76, no. 27: 1-23.

SCOTTI, JURIĆ, R., 1998, Problemi di bilinguismo nel territorio istro-quarnerino, "Studium Educationis, Lineamenti di Didattica", 4, 786-794, Cedam, Padova.

SCOTTI, JURIĆ, R., 2003, Bilinguismo precoce: usi e funzioni linguistiche, Edit, Pietas Iulia, Rijeka.

SCOTTI, JURIĆ, R., AMBROSI, RANDIĆ N., 2010, Italiano L2. Motivazione all'insegnamento/apprendimento della lingua italiana, Edit, Rijeka.

TSCHIRNER, E., 1997, Deutsch als Fremdsprache im Medienzeitalter, „Fremdsprache Deutsch“, Sondernummer 2/1997, 55 - 58.

WARDHAUGH, R., 1995, Szociolingvisztika, Osiris-Századvég, Budapest. 


\section{INTERKULTURALNA KOMUNIKACIJSKA KOMPETENCIJA: PRIMJERI MULTILINGVISTIČKE INTERAKCIJE U ISTRI}

$\mathrm{Na}$ temelju jednoga istraživanja o interkulturalnoj komunikacijskoj kompetenciji u području istarskoga multilingvizma u članku se analizira verbalna komunikacija dvojezičnih mladih kojima je talijanski materinji jezik. Nakon kratkoga opisa istraživanja o dvojezičnosti i multikulturalizma na istarsko-kvarnerskome području, ispitane su motivacije koje donose uporabu code-switchinga i lingvističke interferencije tijekom neformalnih interakcija sudionika u razgovoru. Primjeri snimanih razgovora u obiteljskoj situaciji i drugih - društvenih mreža, analizirani su kako bi se pokazala mogućnost pružanja značajnoga uvida u interkulturalnu komunikaciju i jezik. Posebno se pokušalo objasniti kako izbor jezika i izmjena jezika može ispuniti svrhu komunikacije i kako se ograničena kompetencija u trećem i četvrtom jeziku može rabiti $\mathrm{u}$ interkulturalnoj komunikaciji.

Ključne riječi: interkulturalna kompetencija, interkulturalna komunikacija, dvojezični razgovor, code-switching, interferencija, lingvističke funkcije

\section{INTERCULTURAL COMPETENCE: SOME EXEMPLES OF PLURILINGUISTICS IN ISTRIA}

According to research on intercultural communication within the multilingual zone of Istria, this paper analyses the verbal interactions among bilingual youth whose mother tongue is Italian. Following a brief description of bilingual and multicultural studies in the Istrian-Kvarnerian area, the main motivations which lead to the use of code-switching are analysed, as well as lingual interference during informal interaction among speakers. What is discussed here are examples recorded in familiar situations and those abstracted from a social network in order to demonstrate that the analysis of conversation can provide an template for an overview of intercultural and linguistic communication. Furthermore, this paper attempts to explain how the choice of language and the alternation of language are able to satisfy the pressing purposes of communication and how the opportunity of a limited competence of the third and fourth language is made manifest in intercultural communication.

Key words: intercultural competence, bilingual discourse, code-switching, interference, linguistic function 